\title{
COMMUNICATION
}

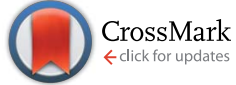

Cite this: RSC Adv., 2015, 5, 73958

Received 1st July 2015

Accepted 24th August 2015

DOI: $10.1039 / c 5 r a 12789 k$

www.rsc.org/advances

\section{Early stages of ZIF-8 film growth: the enhancement effect of primers exposing sulfonate groups as surface-confined nucleation agents $\uparrow$}

\author{
Jimena S. Tuninetti, Matías Rafti and Omar Azzaroni*
}

We describe the use of sulfonate-terminated self-assembled monolayers as very efficient surface-confined nucleation agents for rapid growth of dense, thick and well-percolated ZIF-8 films.

\section{Introduction}

Metal-organic frameworks (MOFs) belong to a relatively new class of hybrid organic-inorganic materials featuring high surface areas, a wide range of pore sizes, pore shapes, and a great variety of chemical functionalities exposed in the pore walls. ${ }^{1-5}$ These interesting features are possible due to an overwhelming number of organic linkers and metal cluster combinations, thus yielding a very broad variety of materials suitable for diverse applications such as; e.g., separations, ${ }^{6,7}$ gas storage ${ }^{\mathbf{8}, 9}$ heterogeneous catalysis, ${ }^{\mathbf{1 0}}$ or biological related uses when biocompatibility is ensured. ${ }^{11-13}$ Zeolitic imidazolate frameworks (ZIFs) constitute a subclass of MOFs, in which the zeolite-type topology (with its proverbial fixed $\mathrm{Si}-\mathrm{O}-\mathrm{Si} 145^{\circ}$ angle) is achieved by the formation of a geometrically similar $\mathrm{M}-\mathrm{N}-\mathrm{M}$ bond; with $\mathrm{N}$ symbolizing a nitrogen atom from an imidazolate or substituted imidazolate linker, and $\mathrm{M}$ a metal ion such as $\mathrm{Co}^{2+}$ or $\mathrm{Zn}^{2+}$ tetrahedrally coordinated. In particular, ZIF-8 is an appealing material for water based applications due to its demonstrated thermal and water stability, its high surface area (approx. 1400-1500 $\mathrm{m}^{2} \mathrm{~g}^{-1}$ ), and well defined $-\mathrm{CH}_{2}$ terminated spherical hydrophobic large pores (inner pore diameter $1.16 \mathrm{~nm})^{\mathbf{1 4}}$ with small pore apertures (pore window size $0.34 \mathrm{~nm}) .{ }^{15}$ The initial step for homogeneous nucleation of a crystalline solid (such as ZIF-8 MOF) from a solution of its precursors can be described as a random

Instituto de Investigaciones Fisicoquímicas Teóricas y Aplicadas (INIFTA) Universidad Nacional de La Plata - CONICET, CC. 16 Suc. 4, 1900 La Plata, Argentina. E-mail: azzaroni@inifta.unlp.edu.ar; Fax: +54 221425 4642; Tel: +54 2214257430

$\dagger$ Electronic supplementary information (ESI) available. See DOI: $10.1039 / \mathrm{c} 5 \mathrm{ra} 12789 \mathrm{k}$ process. ${ }^{16-18}$ This implies that, given that supersaturation conditions are met, critical size nuclei leading to solid formation do not appear immediately; and the time needed for this process to occur is commonly referred as the induction time. If surfaces where heterogeneous nucleation can occur are present, then the induction time can be drastically reduced. Moreover, the use of chemically modified surfaces featuring terminal groups compatible with MOFs building blocks, were reported to have both a strong effect on induction time reduction, and also to cause preferential growth according to a certain crystalline direction. ${ }^{19,20}$ Supported MOFs films are widely used for sensor (usually thin films are desired), ${ }^{21-25}$ and separation applications (e.g., pervaporation membranes, which require relatively thick films). ${ }^{26-28}$ Most common synthetic approaches to create MOF thin films involve layer-bylayer (LbL) growth, secondary growth (SG), and sequential onepot (SOP) growth. In the LbL approach precursor solutions are kept separated, and the substrate is sequentially dipped into linker and metal ion solutions. ${ }^{29-32}$ SG method consists in a pre-synthesis step where MOFs nanocrystals are synthesized and separated. Precursors are then seeded over the chosen support (e.g., via spin-coating), and exposed to a (usually slightly super-saturated) solution containing the precursors. ${ }^{33,34}$ This yields thick films, growing from seeded material acting as heterogeneous nuclei. Usually, cracks can be observed in areas where growing film segments merge (sometimes visible by electron microscopy) causing a reduced control over percolation. On the other hand, sequential onepot (SOP) strategy consists in simply dipping the substrate in a solution of precursors during a preset reaction time and temperature, which will depend on the MOF type. Homogeneous and heterogeneous nucleation processes occur simultaneously, and the (maximum) film thickness can be controlled by the amount of SOP cycles performed. This ultimately constrains the method flexibility, which is a pay-off for the much simpler implementation. ${ }^{29,35}$ It is important to note that SOP can be greatly improved if a surface-confined chemical primer is used to enhance heterogeneous over 
homogeneous nucleation. In this way, additional control over formation kinetics and morphology of the film would be gained. ${ }^{36-41}$ Despite the mentioned exciting developments in this field, the vast majority of ZIF-8 films grown today are exclusively based on the use of amine-terminated surfaces. However, this vision can change if we consider that $\mathrm{Zn}^{2+}$ ions can also be strongly coordinated by sulfonate groups, thus introducing a new variable to control the heterogeneous nucleation of these materials and modulate the film growth. ${ }^{\mathbf{4 2}}$ To the best of our knowledge the use of sulfonate groups as primers for enhancing ZIF-8 thin film growth remains fully unexplored. Herein, we describe for the first time the use of sulfonate-terminated self-assembled monolayers (SAMs) as very efficient nucleating agents enhancing the rapid growth of thick ZIF-8 films.

\section{Results and discussion}

Synthesis of ZIF-8 thin films was accomplished using the SOP approach from methanolic mother solutions at room temperature (see $\mathrm{ESI} \dagger$ file for further details). Surfaces bearing different primers were prepared by chemisorbing SAMs of $\quad N, N, N$-trimethyl(3-mercaptopropyl)-ammonium chloride (NTMAC), cysteamine, and 3-mercapto-1-propanesulfonic acid (MPSA) on gold substrates. To evaluate the influence of these contrasting functional groups on ZIF-8 films growth, in situ monitoring of growth kinetics using quartz crystal microbalance technique was carried out. Fig. 1 displays time evolution of frequency change $(\Delta f)$ for films grown over freshly prepared modified gold surfaces. Remarkable differences can be observed, not only in terms of the mass/thickness of ZIF-8 films grown on the sensor surface, but also regarding the induction times observed, i.e.: the time required to the start of film growth after mixing of precursor solutions. As is well known the time required for triggering heterogeneous nucleation can be reduced if favourable interactions between exposed surface moieties and solvated MOFs precursor exist. The critical role of surface chemistry becomes evident if we compare the induction time for ZIF-8 films grown on bare-Au substrates ( $\sim 35 \mathrm{~min})$ with values obtained for substrates modified with quaternary ammonium (NTMAC, $\sim 15 \mathrm{~min}$ ), primary amine (cysteamine, $\sim 10 \mathrm{~min}$ ) and sulfonate (MPSA, $\sim 10 \mathrm{~min}$ ) functionalities. The relation between induction times for heterogeneous $\left(t_{\mathrm{Het}}\right)$ and homogeneous nucleation $\left(t_{\mathrm{Hom}}\right)$ plays a fundamental role in determining the morphology of the films obtained. Consequently, as $t_{\mathrm{Het}}$ becomes increasingly larger than $t_{\mathrm{Hom}}$, it also increases homogeneous nucleation contribution to film morphology; i.e., growth occur preferentially from seeded particles on the surface, instead of growth from precursors by direct interaction with surface. This in turn leads to less dense and scarcely percolated films. It is important to highlight that, even when similar $t_{\text {Het }}$ values were observed for modified gold QCM sensors, surfaces bearing sulfonate groups exhibited a significant increase in mass. This reveals that the presence of MPSA favours a more extensive film growth, even when comparing with traditionally used primary amine-terminated surfaces (cysteamine-modified). ${ }^{22}$
Fig. 1 inset shows scanning electron microscopy images of films grown on MPSA-modified and bare Au substrates. Films grown on cysteamine-modified surfaces exhibit similar morphological features to films grown on MPSA modified substrates, while NTMAC images are similar those obtained when using bare $\mathrm{Au}$ (not shown, see ESI $\dagger$ ). It is important to note that $-\mathrm{NH}_{2}$ and $-\mathrm{SO}_{3}{ }^{-}$are able to strongly interact with $\mathrm{Zn}^{2+}$ whereas quaternary amines do not interact with any constituent of the MOF architecture. Hence this comparison illustrates the marked difference in growth kinetics when non-chelating and strongly chelating groups are employed to grow the film. It is evident that homogeneous nucleation dictates rhombic dodecahedron morphology observed for bare $\mathrm{Au},{ }^{\mathbf{4 3}}$ while much denser films with less defined morphology were obtained for MPSA modified substrates. Further characterization of films presented in Fig. 1 was carried via powder X-ray diffraction (PXRD). Fig. 2 displays diffractograms obtained for bare and surface modified Au substrates, together with calculated ZIF-8 bulk material diffractogram. It can be observed that only films grown on MPSA- and cysteamine-modified substrates display the main features corresponding to bulk material after just one SOP synthesis cycle, while films grown over bare gold and NTMAC-modified substrates produce weak nearly amorphous diffractograms, in line with the less denser films observed via SEM (see ESI $\dagger$ for diffractogram evolution with increasing

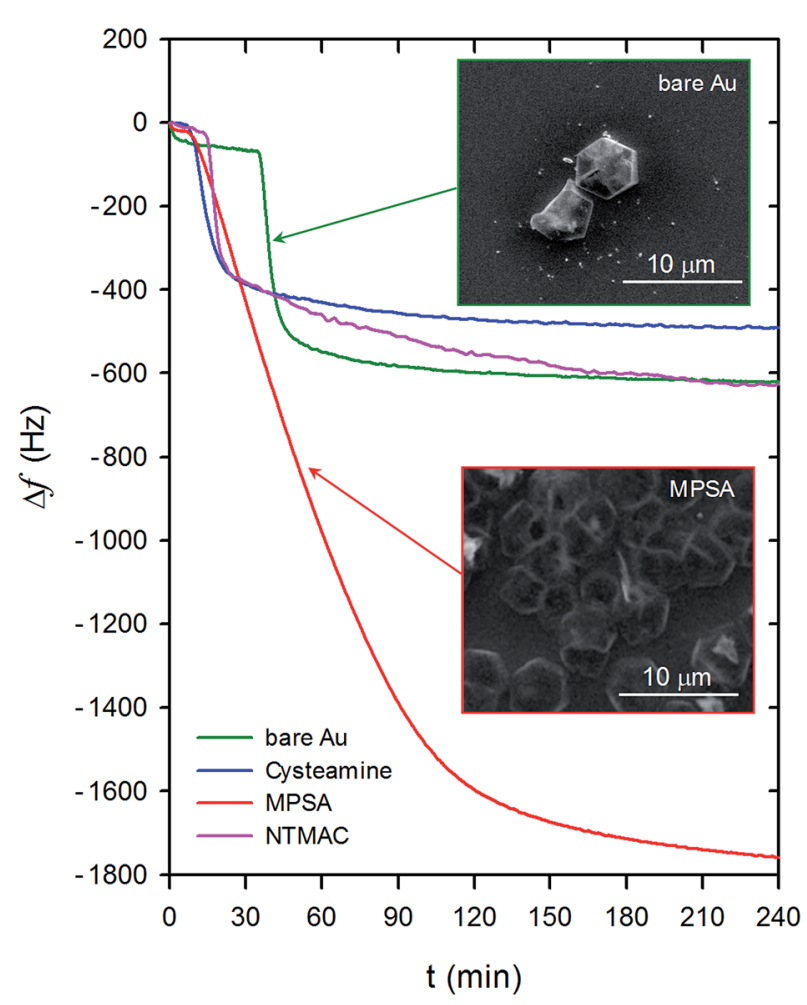

Fig. 1 Deposition of ZIF-8 films on different surfaces, as detected by QCM: (green) bare gold, (blue) cysteamine-modified gold, (red) MPSAmodified-gold, and (turq) NTMAC-modified gold. The mass of the deposited MOF is directly proportional to the frequency change. Insets show SEM images of film morphology after one SOP cycle for MPSA modified and bare gold substrates. 


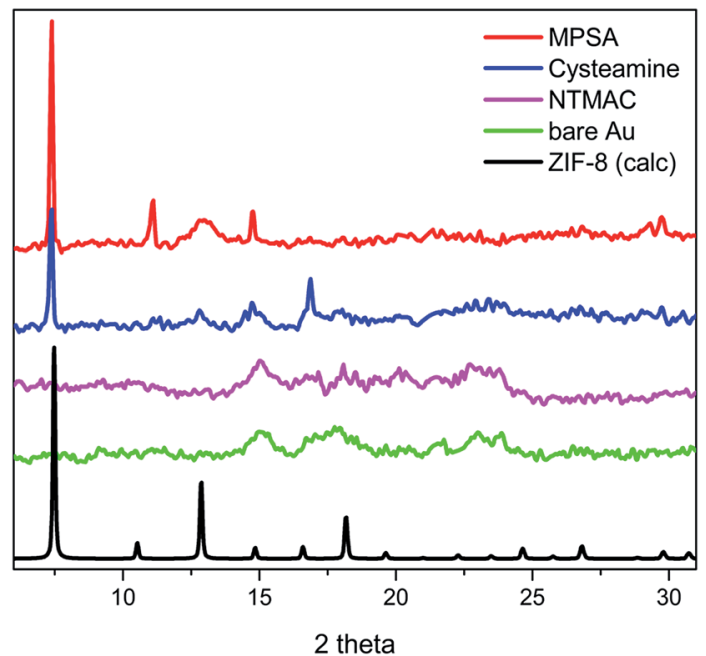

Fig. 2 PXRD patterns for ZIF-8 films grown on different Au modified surfaces corresponding to QCM experiments presented in Fig. 1 (same colour code used). The calculated diffractogram for bulk ZIF-8 material is also depicted (black).

number of SOP cycles). To gain further insight into the nucleation process and the interplay between homogeneous and heterogeneous nucleation, we investigated the ZIF-8 bulk particle size time evolution by dynamic light scattering (DLS). Results presented in Fig. 3 indicate that homogeneous nucleation induction time $\left(t_{\mathrm{Hom}} \sim 5 \mathrm{~min}\right)$ is within the range of previously QCM determined $t_{\mathrm{Het}}$.

Moreover, DLS experiments for hydrodynamic diameter time evolution $\left(D_{\mathrm{H}}\right)$ indicates that heterogeneous nucleation on cysteamine and MPSA-modified Au surfaces takes place in the presence of homogeneously nucleated ZIF-8 particles of $D_{\mathrm{H}} \sim 50$ $\mathrm{nm}\left(t_{\mathrm{Het}} \sim 10 \mathrm{~min}\right)$, whereas in the case of bare gold substrates exhibiting larger induction times, film growth takes places in the presence of particles with $D_{\mathrm{H}}>500 \mathrm{~nm}$. These results

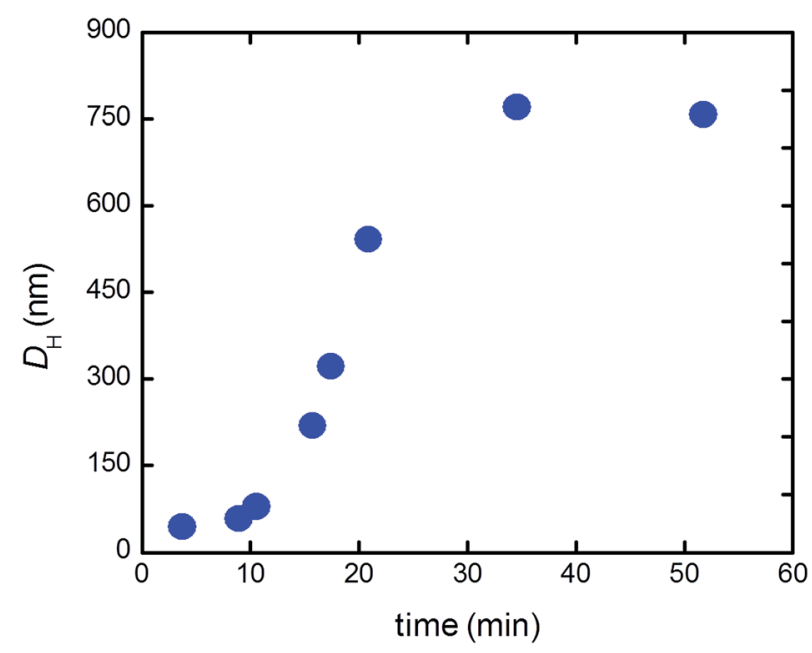

Fig. 3 Temporal evolution of ZIF-8 particle-size (hydrodynamic diameter, $D_{H}$ ) resulting from homogeneous nucleation in methanolic solutions, as determined by DLS. illustrate how important surface chemistry is to control $t_{\mathrm{Het}}$ and, consequently, to avoid undesired side effects from homogeneous nucleation. In addition, the experimental evidence shows that it is possible to introduce drastic changes in the chemical identity of the surface, e.g.: from $-\mathrm{NH}_{2}$ to $-\mathrm{SO}_{3}{ }^{-}$, without significantly altering the induction time for MOF film growth. Film homogeneity depends on the relative importance of heterogeneous and homogeneous growth. If heterogeneous process dominates, then a compact film could be expected; while if the opposite is true, cracks and less dense arrangements will appear. The reduction in $t_{\mathrm{Het}}$ observed for surfaces modified with cysteamine and MPSA suggest that these primers will produce more compact films.

In order to test such hypothesis, permeation experiments were carried using cyclic voltammetry (CV) technique. To assess film percolation and compactness, CV experiments were conducted using the negatively charged $\mathrm{Fe}(\mathrm{CN})_{6}{ }^{3-} / \mathrm{Fe}(\mathrm{CN})_{6}{ }^{4-}$ redox probe. The inherent microporosity of ZIF-8 material features pore windows of $\sim 0.34 \mathrm{~nm}$ width, which are not large enough to fit the redox probe. Due to this fact, $\mathrm{CV}$ experiments can be employed to evaluate the presence of loosely packed films with large intergrain mesoporosity as well as the presence of dense films in which grains are tightly packed. Given the hydrophobic nature of the ZIF-8 material, and the relative sizes of redox probe used and micropores; the permeation behavior can be rationalized in terms of the effective assembly of the ZIF-8 grains within the film. Dense arrangements of grains should lead to scarce or none intergrain mesoporosity, while the contrary presumption applies for less dense films; this would result in low and high electrochemical signals of the redox probe, respectively. As expected, the results obtained show that the films synthesized on quaternary amine-modified or bare gold surfaces are highly permeable to the redox probe. The CV results suggest that full percolation of surface-confined grains was not achieved for films grown on NTMAC-modified and bare Au surfaces (Fig. 4). On the other hand, MPSA- and cysteamine-

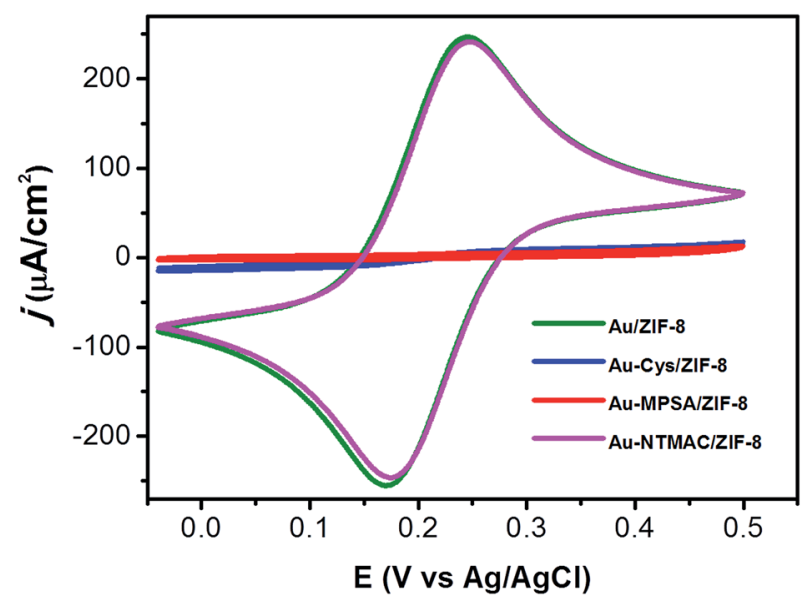

Fig. 4 Voltammetric response of electrodes constituted of ZIF-8 films grown on different chemically-modified conducting substrates: (green) bare gold, (blue) cysteamine-, (red) MPSA-, and (turq) NTMACmodified gold substrates; in the presence of $1 \mathrm{mM} \mathrm{Fe}(\mathrm{CN})_{6}{ }^{3-}$ / $\mathrm{Fe}(\mathrm{CN})_{6}{ }^{4-}+100 \mathrm{mM} \mathrm{KCl}$ (aqueous solution). 
modified surfaces displayed a much lower electrochemical signal, which can, in turn, be related to the presence of rather compact films precluding the passage of the redox probes through the MOF layer (Fig. 4).

\section{Conclusions}

An important requirement in the preparation of interfacial architectures based on MOFs is devising strategies to control their nucleation and growth. Over the last years, sequential onepot growth of ZIF-8 thin films has been mostly accomplished using amine-terminated surfaces as primers. But it remains a practical challenge to manipulate the chemical nature of the substrate in order to enhance the growth process without altering the structural properties of the synthesized films. In this work we showed for the first time the use of sulfonateterminated primers as efficient surface-confined nucleating agents for rapid growth of ZIF-8 MOF.

We observed that favourable interactions between solvated MOF precursors and surface exposed moieties are crucial to manipulate the dynamics of film growth. It was demonstrated that under comparable conditions, MPSA-modified surfaces promote a remarkable $\sim 3.5$ fold increase in $\Delta f$ values (and therefore on the amount of material deposited) as compared with cysteamine-modified substrates without altering the induction time for heterogeneous nucleation. Remarkably, PXRD of films obtained after just one SOP cycle over $\mathrm{SO}_{3}{ }^{-}$modified and (to a lesser extent) $\mathrm{NH}_{2}$-terminated surfaces, already display ZIF-8 bulk material main features. In line with these findings, CV experiments provide evidence of a clear difference between MPSA- and cysteamine-modified well percolated films with low intergrain mesoporosity, versus bare $\mathrm{Au}$ and NTMAC-modified non-percolated films. From a synthetic viewpoint, introducing new primers is a particularly interesting strategy to optimize the growth of MOF thin films. Much more important - and also technologically relevant- as our array of synthetic strategies grows, so does our repertoire to "engineer" thin film architectures using metal-organic frameworks as active and functional building blocks. In this context, we believe that these results will further broaden the range of possibilities to design MOF-based hybrid materials as well as heterosupramolecular architectures.

\section{Experimental}

$\mathrm{Zn}\left(\mathrm{NO}_{3}\right)_{2} \cdot 6 \mathrm{H}_{2} \mathrm{O}, \quad$ 2-methyl-imidazole, and 3-mercapto-1propanesulfonic acid, sodium salt (MPSA) were purchased from Sigma Aldrich. 2-Aminoethaenethiol hydrochloride (cysteamine) was purchased from Acros Organics. All reagents were used as received. The $N, N, N$-trimethyl(3-mercaptopropyl) ammonium chloride was synthesized as described in literature. $^{44}$

\section{Film synthesis and surface modification of Au substrates}

ZIF-8 films were synthesized over chemically modified Au substrates using methanolic solutions of precursors according to previously published procedure. ${ }^{45}$ Briefly, it consists on the following steps: (i) Au substrate was modified using solutions of the selected alkanethiol (concentrations used were $5 \mathrm{mM}$ cysteamine in ethanol, $1 \mathrm{mM}$ NTMAC in ethanol, and $20 \mathrm{mM}$ MPSA in $10 \mathrm{mM} \mathrm{H}_{2} \mathrm{SO}_{4}$ ) at room temperature overnight. Treated substrates were, then rinsed, and dryed with $\mathrm{N}_{2}$, (ii) clean modified $\mathrm{Au}$ substrates were immersed in a fresh mixture of $\mathrm{Zn}\left(\mathrm{NO}_{3}\right)_{2} \cdot 6 \mathrm{H}_{2} \mathrm{O}(10 \mathrm{~mL}, 25 \mathrm{mM})+2$-methyl-imidazole $(10 \mathrm{~mL}$, $50 \mathrm{mM}$ ) stock solutions for appropriate time according to the primer used (see Section 3) at room temperature, (iii) substrates whereas then rinsed with fresh methanol and dried with $\mathrm{N}_{2}$ prior to the next cycle.

\section{Film characterization}

ZIF-8 film growth over bare and modified $\mathrm{Au}$ substrates was followed using a Quartz Crystal Microbalance (QCM 200) from Stanford Research Systems. For post-synthesis characterization morphology of the films was studied by scanning electron microscopy (ESEM FEI-Quanta 200) and powder X-ray diffraction (Phillips X'Pert X-Ray Diffractometer); size analysis were assessed by DLS using a Zetasizer Nano ZS from Malvern.

\section{Cyclic voltammetry experiments}

Cyclic voltammetry experiments were carried out using a Reference 600 potentiostat from Gamry, with a three-electrode setup. $\mathrm{Ag} / \mathrm{AgCl}$ and $\mathrm{Pt}$ wire were used as reference electrode and counter electrodes, respectively. In all the electrochemical experiments $1 \mathrm{mM} \mathrm{Fe}(\mathrm{CN})_{6}{ }^{3-} / \mathrm{Fe}(\mathrm{CN})_{6}{ }^{4-}+100 \mathrm{mM} \mathrm{KCl} \mathrm{solu-}$ tions were used.

\section{Acknowledgements}

J.T gratefully acknowledges CONICET for a postdoctoral fellowship. This work was supported by CONICET (PIP 11220130100370CO) ANPCyT (PICT-2010-2554 and PICT-20130905). M.R. and O.A. are CONICET fellows.

\section{Notes and references}

1 G. Férey, Chem. Soc. Rev., 2008, 37, 191-214.

2 S. T. Meek, J. A. Greathouse and M. D. Allendorf, Adv. Mater., 2011, 23, 249-267.

3 M. O'Keeffe and O. M. Yaghi, Chem. Rev., 2012, 112, 675-702.

4 H. Furukawa, K. E. Cordova, M. O'Keeffe and O. M. Yaghi, Science, 2013, 341, 974.

5 S. Furukawa, J. Reboul, S. Diring, K. Sumida and S. Kitagawa, Chem. Soc. Rev., 2014, 43, 5700-5734.

6 J. R. Li, J. Sculley and H. C. Zhou, Chem. Rev., 2012, 112, 869932.

7 R. Ahmad, A. G. Wong-Foy and A. J. Matzger, Langmuir, 2009, 25, 11977-11979.

8 G. Férey, C. Serre, T. Devic, G. Maurin, H. Jobic, P. L. Llewellyn, G. de Weireld, A. Vimont, M. Daturi and J.-S. Chang, Chem. Soc. Rev., 2011, 40, 550-562.

9 S. Bureekaew, S. Shimomura and S. Kitagawa, Sci. Technol. Adv. Mater., 2008, 9, 014108. 
10 J. Gascon, A. Corma, F. Kapteijn and F. X. Llabrés i Xamena, ACS Catal., 2014, 4, 361-378.

11 P. Horcajada, T. Chalati, C. Serre, B. Gillet, C. Sebrie, T. Baati, J. F. Eubank, D. Heurtaux, P. Clayette, C. Kreuz, J.-S. Chang, Y. K. Hwang, V. Marsaud, P.-N. Bories, L. Cynober, S. Gil, G. Férey, P. Couvreur and R. Gref, Nat. Mater., 2010, 9, 172-178.

12 L. Cooper, T. Hidalgo, M. Gorman, T. Lozano-Fernández, R. Simón-Vázquez, C. Olivier, N. Guillou, C. Serre, C. Martineau, F. Taulelle, D. Damasceno-Borges, G. Maurin, Á. González-Fernández, P. Horcajada and T. Devic, Chem. Commun., 2015, 51, 5848-5851.

13 J. G. Yañuk, M. L. Alomar, M. M. Gonzalez, F. Simon, R. ErraBalsells, M. Rafti and F. M. Cabrerizo, Phys. Chem. Chem. Phys., 2015, 17, 12462-12465.

14 K. Zhang, R. P. Lively, M. E. Dose, A. J. Brown, C. Zhang, J. Chung, S. Nair, W. J. Koros and R. R. Chance, Chem. Commun., 2013, 49, 3245-3247.

15 X. C. Huang, Y. Y. Lin, J. P. Zhang and X. M. Chen, Angew. Chem., Int. Ed., 2006, 45, 1557-1559.

16 N. Pienack and W. Bensch, Angew. Chem., Int. Ed., 2011, 50, 2014-2034.

17 D. Erdemir, A. Y. Lee and A. S. Myerson, Acc. Chem. Res., 2009, 42, 621-629.

18 D. Knezic, J. Zaccaro and A. S. Myerson, J. Phys. Chem. B, 2004, 108, 10672-10677.

19 M. C. McCarthy, V. Varela-Guerrero, G. V. Barnett and H. K. Jeong, Langmuir, 2010, 26, 14636-14641.

20 V. Stavila, J. Volponi, A. M. Katzenmeyer, M. C. Dixon and M. D. Allendorf, Chem. Sci., 2012, 3, 1531-1540.

21 J. Yao and H. Wang, Chem. Soc. Rev., 2014, 43, 4470-4493.

22 A. Bétard and R. A. Fischer, Chem. Rev., 2012, 112, 10551083.

23 P. Horcajada, C. Serre, D. Grosso, C. Boissière, S. Perruchas, C. Sanchez and G. Férey, Adv. Mater., 2009, 21, 1931-1935.

24 D. Bradshaw, A. Garai and J. Huo, Chem. Soc. Rev., 2012, 41, 2344.

25 L. Heinke and C. Woll, Phys. Chem. Chem. Phys., 2013, 15, 9295-9299.

26 L. Diestel, H. Bux, D. Wachsmuth and J. Caro, Microporous Mesoporous Mater., 2012, 164, 288-293.
27 S. Hermes, F. Schröder, R. Chelmowski, C. Wöll and R. A. Fischer, J. Am. Chem. Soc., 2005, 127, 13744-13745.

28 Y. S. Li, H. Bux, A. Feldhoff, G. N. Li, W. S. Yang and J. Caro, Adv. Mater., 2010, 22, 3322-3326.

29 K. Kida, K. Fujita, T. Shimada, S. Tanaka and Y. Miyake, Dalton Trans., 2013, 11128-11135.

30 O. Shekhah, L. Fu, R. Sougrat, Y. Belmabkhout, A. J. Cairns, E. P. Giannelis and M. Eddaoudi, Chem. Commun., 2012, 48, 11434.

31 O. Shekhah, Materials, 2010, 3, 1302-1315.

32 M. C. So, S. Jin, H. J. Son, G. P. Wiederrecht, O. K. Farha and J. T. Hupp, J. Am. Chem. Soc., 2013, 135, 15698-15701.

33 F. Tian, A. M. Cerro, A. M. Mosier, H. K. Wayment-Steele, R. S. Shine, A. Park, E. R. Webster, L. E. Johnson, M. S. Johal and L. Benz, J. Phys. Chem. C, 2014, 118, 14449-14456.

34 D. Jiang, A. D. Burrows, R. Jaber and K. J. Edler, Chem. Commun., 2012, 48, 4965-4967.

35 S. Li, W. Shi, G. Lu, S. Li, S. C. J. Loo and F. Huo, Adv. Mater., 2012, 24, 5954-5958.

36 J. Liu, F. Sun, F. Zhang, Z. Wang, R. Zhang, C. Wang and S. Qiu, J. Mater. Chem., 2011, 21, 3775.

37 O. Shekhah, H. Wang, D. Zacher, R. A. Fischer and C. Wöll, Angew. Chem., Int. Ed., 2009, 48, 5038-5041.

38 D. Zacher, R. Schmid, C. Wöll and R. A. Fischer, Angew. Chem., Int. Ed., 2011, 50, 176-199.

39 J. Liu, O. Shekhah, X. Stammer, H. K. Arslan, B. Liu, B. Schüpbach, A. Terfort and C. Wöll, Materials, 2012, 5, 1581-1592.

40 A. Schoedel, C. Scherb and T. Bein, Angew. Chem., Int. Ed., 2010, 49, 7225-7228.

41 J. J. Gassensmith, P. M. Erne, W. F. Paxton, C. Valente and J. F. Stoddart, Langmuir, 2011, 27, 1341-1345.

42 R. Zhang, S. Ji, N. Wang, L. Wang, G. Zhang and J. R. Li, Angew. Chem., Int. Ed., 2014, 53, 9775-9779.

43 J. Cravillon, C. A. Schröder, H. Bux, A. Rothkirch, J. Caro and M. Wiebcke, CrystEngComm, 2012, 14, 492.

44 J. Tien, A. Terfort and G. M. Whitesides, Langmuir, 1997, 13, 5349-5355.

45 C. Hou, Q. Xu, J. Peng, Z. Ji and X. Hu, ChemPhysChem, 2013, 14, 140-144. 